\title{
A Beacon-based Approach for RF Source localization in Outdoor NLOS Environment for Search and Rescue Missions
}

\author{
Muhammad Hassan Fares ${ }^{1,2}$, Hadi Moradi ${ }^{1,3, *}$, \\ Mahmoud Shahabadi ${ }^{4}$ and Yasser Mohanna ${ }^{5}$ \\ ${ }^{1}$ Advanced Robotics and Intelligent Systems Laboratory, School of ECE, University of Tehran, Iran \\ ${ }^{2}$ EDST, Lebanese University, Hadath, Lebanon \\ ${ }^{3}$ Adjunct Research Prof, ISRI, SKKU, South Korea \\ ${ }^{4}$ School of ECE, University of Tehran, Iran \\ ${ }^{5}$ Department of Physics and Electronics, Lebanese University, Hadath, Lebanon
}

('Corresponding author's e-mail: moradih@ut.ac.ir)

Received: 8 February 2021, Revised: 8 June 2021, Accepted: 10 June 2021

\begin{abstract}
Due to its low implementation cost, the combination of the Received Signal Strength (RSS) with the Angle of Arrival (AOA) measurements is one of the solutions for Radio Frequency (RF) source localization, especially in a Non-Line of Sight (NLOS) environment. It is critical to determine the search space for a person who is lost in rural areas where the mobile network is unavailable due to a lack of Base Tower Stations (BTS) in order to reduce search time. In this paper, we introduce a new beacon-based approach for RF source localization, where the RF signal is received in NLOS after 1-bounce reflection, by combining the information coming from both the RSS-AOA sensors and the beacons, which are used as helpers- that move along a determined path. The proposed approach relies on determining the reflector's pose first, after which the RF source is localized. The work has been verified in simulation and the Root Mean Square Error (RMSE) is used as a performance metric for RF source localization. Results show that our proposed approach has the lowest RMSE among localization methods mentioned in the literature under the same conditions.
\end{abstract}

Keywords: Beacon-based approach, NLOS environment, RF source localization, Rural areas, Search and rescue missions

\section{Introduction}

Localization has broad civilian and military applications, such as search and rescue missions $[1,2]$. Nowadays, mobile phones have become a major medium of communications. Since they are used by everyone, then their known signals' power can be used in target localization missions [3]. When a mobile phone is out of service, its signal drops out, and the classical mobile phone localization methods based on BTS towers such as the Enhanced-Lateration technique, introduced in [4], cannot be used. Therefore, researchers proposed new approaches for search and rescue missions in outdoor environments, based on the RF source's signals, as introduced in [5,6]. In such cases, time is critical, and S\&R missions may become worthless and futile [7].

When the target's signal is received directly, the localization can be performed using some methods such as Time of Arrival (TOA), Time Difference of Arrival (TDOA), Received Signal Strength (RSS) and Angle of Arrival (AOA). For instance, the authors in [8] show that 3 TOA receivers are enough to determine the target position in 2-D space in LOS condition, based on the tri-lateration method. Moreover, TDOA is used with the given that the receivers are synchronized, which is hard to implement $[9,10]$.

Due to the low implementation cost, RSS is widely used as one of the localization methods; it measures the strength of the coming signal, where the distance from the RF source to the receiver can be calculated, assuming the known signal's frequency. Another method is DOA. It is based on the direction of the coming signal, wherein 2 receivers are needed to determine the intersection point from the bearings toward the target. Although the mentioned methods are used for LOS RF source localization with high accuracy, they are considered less efficient methods for an NLOS environment due to the errors coming 
from the reflector(s) between the RF source and the receiver. As a result of the blocking of the LOS between the RF source and the receiver, this problem is regarded as a difficult one. The RF source localization in the NLOS environment is introduced in [11-14], in which the authors proposed many methods to handle the problem. For that issue, methods can be divided into 2 major categories. The first one is a combination of 2 or more traditional methods, such as the combination of AOA and RSS $[15,16]$. The other is shown in [16] where the authors propose a hybrid TOA/AOA cooperative localization for NLOS RF sources.

Beacon-based approaches are the other category of localization methods, especially for the NLOS environment [17-21]. In localization missions, beacons act as helpers which are aware of their positions; they transmit signals with known power, used in comparison to the signal received from the RF source that should be localized. In such conditions, the number of beacons and their types should be determined, in addition to their placements if stationary, or their paths if moving.

In recent decades, beacons have been widely used as helpers in localization problems, especially in the NLOS environment. For instance, each member of the rescue team can act as a beacon with a known position aware of an RF source helping in the localization of the target and reflector. The challenge is to minimize the number of needed beacons to localize both the reflector and RF source, with high accuracy. In addition, beacon placement should be taken into consideration due to the high number of beacons needed to cover a large search space. In some papers, authors propose a determined number of fixed beacons for localizing a target [19-21]. However, other dissertations propose the use of moving beacons [22-24]. For localizing a target using beacons, the type of needed beacons and their numbers are related to the area of the search environment. For instance, random beacons can be installed in a small search area or for indoor localization. However, for outdoor localization, the number of needed beacons grows exponentially. For small and indoor areas, Unmanned Aerial Vehicles (UAVs) that can be moving beacons cannot be easily used.

The difference between our proposed approach and other methods, such as the method introduced in [25], is that we have only 1 receiver, and the RF source is localized using 1 observation. However, in that paper, the authors supposedly used 3 receivers to localize the RF source.

The main contribution of this paper is to propose a new RF source localization approach for the NLOS environment, using a single receiver and 2 moving beacons. By using a stationary receiver that rotates to determine the RF source signal's bearing, the distance from the RF source to the receiver is determined based on the information coming from the RSS sensor, assuming an appropriate path loss model. In such a scenario, the search space is determined, in which its perimeter is a circle with the receiver's pose as its center, and the distance from the receiver to the RF source as its radius. Two moving beacons are needed in which their paths are determined w.r.t the search space, as described in the localization approach section. When beacons move, there exist unique positions in which they should be received by the same receiver in the same angle of arrival as the RF source's signal, assuming that no reflectors/obstacles exist between the RF source and beacons.

The rest of the paper is organized as follows. In section 2, we introduce the NLOS RF source localization issue in an outdoor environment. Then, we propose a new beacon-based approach for determining the search space for R\&S missions. Section 3 shows our localization approach in detail in which the beacons' paths are determined. In the result section, we evaluate our proposed method, comparing it with existing methods, and finally, we present a conclusion of our findings.

\section{Problem formulation}

We consider that an RF source's signal is received in NLOS condition, after 1 bounce reflection, through an unknown reflector in an outdoor area. The known power of the RF signal is received by an RSS receiver, in which the signal's bearing is determined using an AOA sensor. From RSS, the distance to the RF source is determined, assuming a proper path loss model. Hence, the distances from the RF source to the receiver and the AOA of the received RF's signal are now known. To decrease the search time, the determination of the search space is the first step of the search mission to locate the lost person (through the equipped RF source's signal) as fast as possible.

In the 2-D space, the RF source and the receiver are described by $\widehat{\mathbf{X}}_{R F}=\left(\hat{x}_{R F}, \hat{y}_{R F}\right)^{T}$ and $\mathbf{X}_{\text {rec }}=$ $\left(x_{r e c}, y_{r e c}\right)^{T}$ respectively. Based on the calculated distance from the RF source to the receiver noted by $\hat{d}_{r e c-R F}$, the search space is determined; it is a circle of center $\mathbf{X}_{r e c}$ and radius $\hat{d}_{r e c-R F}$. For the extremum conditions, i.e., when the reflector is near the receiver or the RF source, which is approximately a LOS condition, the RF source is supposed to be at the perimeter of the search space. Nevertheless, with the reflector noted by $\widehat{\mathbf{X}}_{r e f}=\left(\hat{x}_{r e f}, \hat{y}_{r e f}\right)^{T}$, the RF source is inside the search area as seen in Figure 1. 
Table 1 Important notations used in this paper.

\begin{tabular}{ll}
\hline \multicolumn{1}{c}{ Symbol } & \multicolumn{1}{c}{ Definition } \\
\hline$\widehat{\mathrm{X}}_{R F}$ & The RF source's position \\
$\widehat{\mathrm{X}}_{r e f}$ & The position of the reflector that reflects the RF source's signal \\
$\mathrm{X}_{r e c}$ & The receiver's position/sensor \\
$\alpha$ & The RF signal's bearing received by the receiver \\
$\hat{\theta}$ & The reflector's orientation \\
$\hat{d}_{\text {rec-ref }}$ & The distance between the receiver and the reflector that reflects the RF's signal \\
$\hat{d}_{r e f-R F}$ & The distance between the reflector and the RF source \\
$\mathrm{X}_{\text {bea1 }}$ & The first beacon's position \\
$\mathrm{X}_{\text {bea } 2}$ & The second beacon's position \\
$\varnothing$ & angle of diffraction of the RF source's signal in rad \\
\hline
\end{tabular}

Here, using beacons, the RF source can be localized. The challenge is to determine the needed beacons and their positions (if stationary) or their paths (if moving), where they act as helpers aware of their positions; the proposed approach is introduced in the third section.

For the convenience of readers, the notations used in this paper are summarized in Table 1. In that table, some symbols are known a priori, however, the other variables that should be determined are noted with "A" above them. The subscripts of the distances noted by $d_{i-j}$ describe the distance from $i$ to $j$, respectively.

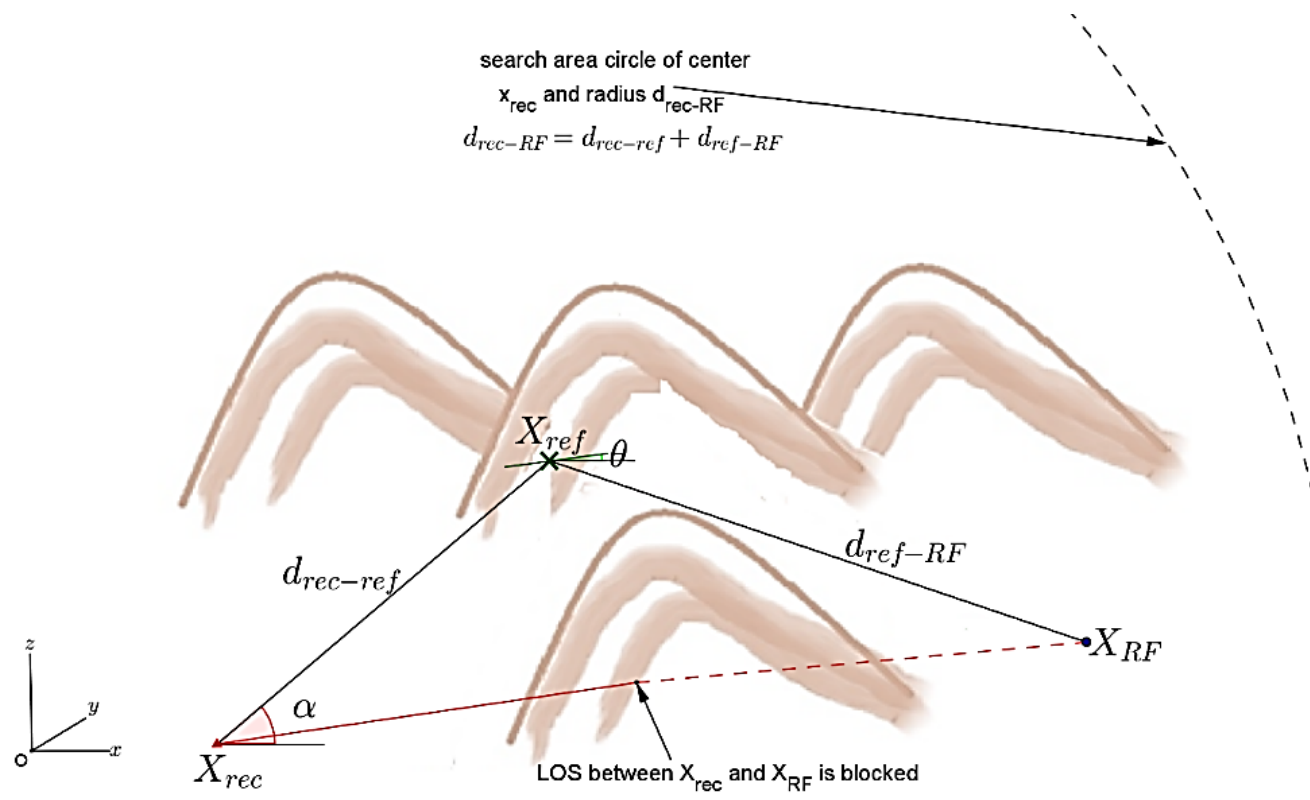

Figure 1 Since the LOS between the receiver and the RF source is blocked due to the existence of an obstacle, the signal coming from the RF source $\left(\widehat{\mathrm{X}}_{R F}\right)$ is received by the receiver sitting at $\mathrm{X}_{\text {rec }}$ through the reflector $\widehat{X}_{r e f}$ (after 1-bounce reflection), which is considered a perfect reflector. The angle of observation of the RF source's signal is $\alpha$, and the angle of the reflector is $\theta$. The distance between the RF source and the receiver (i.e., $\hat{d}_{r e c-R F}$. ) is divided into 2 parts $\left(\hat{d}_{r e c-r e f}\right.$. and $\hat{d}_{r e f-R F}$.), thus, the search space, in which the target is in, is the circle of center $\mathrm{X}_{r e c}$ and radius $\hat{d}_{r e c-R F}$. 


\section{Localization approach}

For a known power of the RF source's signal, the difference between the received power and the transmitted one leads to the signal losses is determined by:

$P_{t}-P_{r}=$ Loss $_{\text {total }}$

in an NLOS environment, the RF signals are under multipath and shadowing influences, in which they propagate after reflection, diffraction, penetration, and scattering. In these conditions, the receiver receives different signals from different orientations at different times. All these losses are introduced in $[5,26]$ and the total loss can be determined by:

$s_{\text {total }}=P L+P D+e(\sigma)$

where PL is the path loss, DL is the diffraction loss, and $e(\sigma)$ can be modeled as a Gaussian random variable with normal standard deviation $\sigma$ to approximate the effects of shadowing, multipath fading and penetration.

For path loss $(P L)$, many models are proposed [27], one of them is:

$P L=32.45+20 \log (f)+20 \log \left(\hat{d}_{r e c-R F}\right)$

in which $f$ is the frequency of the transmitted signal in $\mathrm{MHz}$, and $\hat{d}_{r e c-R F}$ is the distance from the RF source to the receiver in $\mathrm{km}$. Due to the effects of reflection model, $|\psi|$ can be added to (3); it describes the signal absorption value; it is from 0 (if the signal is totally absorbed) to 1 (if the signal is fully reflected):

$P L=32.45+20 \log (f)+20 \log \left(\hat{d}_{r e c-R F}\right)-20 \log |\psi|$

the model of DL can be determined from [5]:

$D L=6.9+20 \log \left(\sqrt{(b-1)^{2}+1}+\mathrm{b}-1\right)$

in which

$b=2.583 \emptyset \sqrt{\frac{f \hat{d}_{r e c-r e f} \hat{d}_{r e f-R F}}{\hat{d}_{r e c-R F}}}$

in Eq.(6), $\varnothing$ is the angle of diffraction in rad, $\hat{d}_{r e c-r e f}$ describes the distance from the receiver to the reflector and $\hat{d}_{r e c-R F}$ is the distance from the reflector to the RF source, in which:

$\hat{d}_{r e c-R F}=\hat{d}_{\text {rec-ref }}+\hat{d}_{\text {ref-RF }}$

the diffraction angle $\emptyset$ (in rad) depends on reflector height, it is calculated by:

$\emptyset=\frac{h_{\boldsymbol{X}_{r e f}}-h_{\boldsymbol{X}_{R F}}}{\hat{d}_{r e f-R F}}+\frac{h_{\boldsymbol{X}_{r e f}}-h_{\boldsymbol{X}_{r e c}}}{\hat{d}_{r e c-r e f}}$

in which $h_{\boldsymbol{X}_{\text {ref }}}$ is the height of reflector, and $h_{\boldsymbol{X}_{R F}}$ and $h_{\boldsymbol{X}_{r e c}}$ are the heights of the RF source and the receiver, respectively. 
For 1 bounce reflection, where the RF signal is received after a single reflection, the distance from the receiver to the RF source, i.e., $\hat{d}_{r e c-R F}$, can be concluded using (4). Thus, the reflector should be placed along the observation line for a moving beacon that moves along the perimeter of the search space (as shown in Figure 2, the search space is the circle of center $\mathrm{X}_{r e c}$ and radius $\hat{d}_{r e c-R F}$ ), and it should be received by the same receiver $\mathrm{X}_{r e c}$ in the same direction of the RF source. When the beacon's signal is received, its position is determined $\left(\mathrm{X}_{\text {bea } 1}\right)$. Another beacon moves along a circle with the same center (i.e., $\mathrm{X}_{r e c}$ ) but a different radius, let's say $R=\hat{d}_{r e c-R F}+d$, where $d$ is chosen randomly. The same receiver detects the other beacon in another position, i.e., $\mathrm{X}_{\text {bea2 }}$. Therefore, the line formed by these beacons intersects with the observation line and leads to the true position of the reflector.

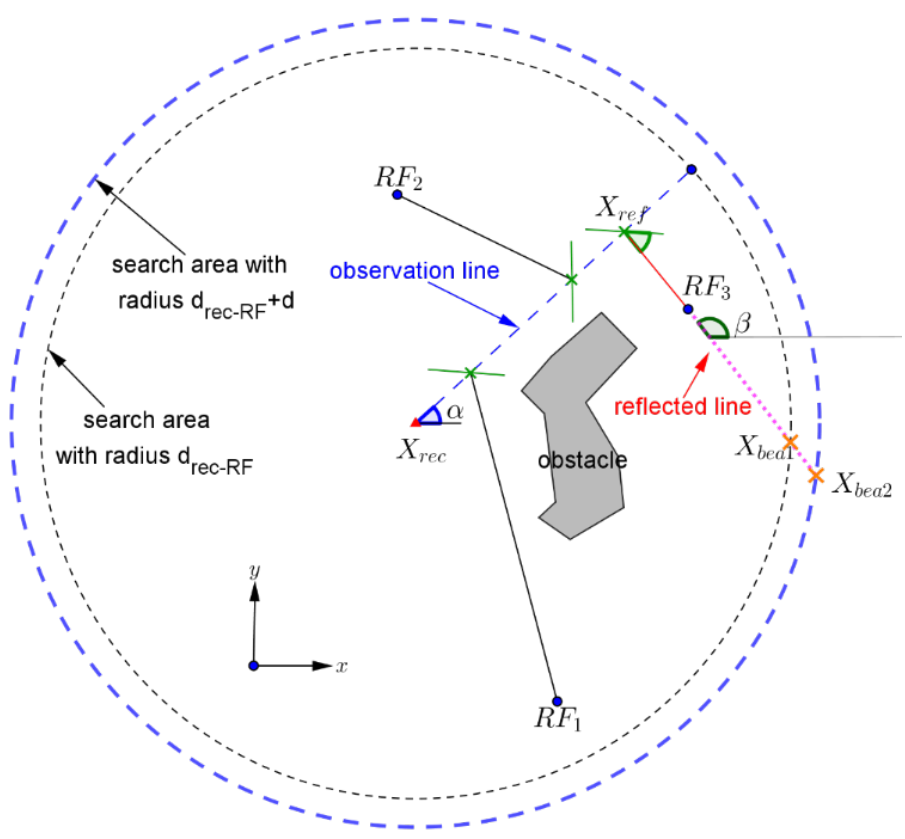

Figure 2 When the beacons $X_{\text {bea } 1}$ and $X_{\text {bea } 2}$ signals are received by the same receiver $X_{r e c}$, the reflected line is determined. The intersection of the reflected line with the observation line leads us to determine the reflector pose. Since there is a 1-bounce reflection, the RF source is localized, assuming that its distance from the receiver is determined using a proper path loss model.

The observation line is determined by the position of the receiver and the angle of arrival of the RF source's signal, in which its equation is described in (9):

$l_{\text {rec-ref }}: y=\tan (\alpha) x+y_{\text {rec }}-x_{\text {rec }} \tan (\alpha)$

the line formed by the 2 beacons is the reflected line, and described by:

$l_{\text {rec-ref }}: \frac{y-y_{\text {bea } 1}}{y_{\text {bea } 2}-y_{\text {bea } 1}}=\frac{x-x_{\text {bea } 1}}{x_{\text {bea } 2}-x_{\text {bea } 1}}$

in which $\mathrm{X}_{\text {bea1 }}=\left(x_{\text {bea } 1}, y_{\text {bea } 1}\right)^{T}$ and $\mathrm{X}_{\text {bea } 2}=\left(x_{\text {bea } 2}, y_{\text {bea } 2}\right)^{T}$ are the coordinates of the 2 beacons when received by the receiver in the world frame.

As shown in Figure 2, the intersection of $l_{\text {rec-ref }}$ and $l_{\text {rec-RF }}$ leads to the reflector's placement. In that figure, for 1-bounce reflection, the reflector is along the observation line. The $R F$ source $R F_{1}, R F_{2}$ and $R F_{3}$ are examples of possible positions of the true RF source, where all these RF sources' positions satisfy the conditions of the AOA's RF signal (i.e., $\alpha$ ) and the total distance between the RF source and the receiver (i.e., $\hat{d}_{r e c-R F}$ ). Suppose that the true position of the RF source that should be localized is $R F_{3}$, then the beacons at positions $\mathrm{X}_{\text {bea1 }}$ and $\mathrm{X}_{\text {bea } 2}$ that are received in the same direction as the RF source 
form a line (the red line in Figure 2). Thus, the reflector $\widehat{\mathrm{X}}_{r e f}$ is determined by the intersection of the reflected line and the observation line. Knowing the reflector leads us to localize the RF source using Eqs. 12(a) - 12(b).

As shown in Figure 2, the orientation of the reflector, i.e., $\hat{\theta}$, can be determined when the reflected line's orientation is determined $(\beta)$. The reflected line's orientation is deduced from the bearing of the line stretching along the 2 beacon's points, with respect to the $\mathrm{x}$-axis, where $\beta=180-(2 \hat{\theta}-\alpha)$, then:

$\hat{\theta}=\frac{180-(\beta-\alpha)}{2}$

thus, the position of the RF source is determined by:

$\hat{x}_{R F}=x_{r e c}+\hat{d}_{r e c-r e f} \cos (\alpha)+\hat{d}_{r e f-R F} \cos (\beta)$

$\hat{y}_{R F}=y_{r e c}+\hat{d}_{\text {rec-ref }} \sin (\alpha)+\hat{d}_{r e f-R F} \sin (\beta)$

with $\hat{d}_{\text {rec-ref }}=\sqrt{\left(\hat{x}_{\text {ref }}-x_{\text {rec }}\right)^{2}+\left(\hat{y}_{\text {ref }}-y_{\text {rec }}\right)^{2}}$ is the distance from the receiver to the reflector, and $\hat{d}_{r e f-R F}=\hat{d}_{r e c-R F}-\hat{d}_{\text {rec-ref }}$ is the distance from the reflector to the RF source.

It is important to notice that the fading of the RF signal is due to multiple reflections. However, the proposed approach can be used for multiple reflections issues, in which the distance between the RF source and the beacon is determined. The same localization concept is applied, but the reflectors cannot be localized. The distance and the orientation of the RF source w.r.t the beacons are determined, and the RF source is localized.

\section{Results and discussion}

In the simulation, our measurements are the angle of arrival of the signal, i.e. $\alpha$ taken from the AOA sensor, and the distance between the RF source and the receiver, i.e. $\hat{d}_{r e c-R F}$ which is determined from the path loss model described in Eqs. (1) - (6). The values of the measurements are chosen randomly and the standard deviations $\sigma_{\hat{d}_{r e c-R F}}$ and $\sigma_{\alpha}$ for $\hat{d}_{r e c-R F}$ and $\alpha$, respectively, are performed differently for each run.

Because of the complicated terrain of rural areas, the RSS and AOA measurements are assumed to be inaccurate enough. However, the main idea of the work is to determine the distance between the RF source and the receiver and the AOA of the RF signal. Hence, the noises of the RSS and AOA measurements are applied in the distance and angle calculations. Considering 0 mean Gaussian noises with variances $v\left(\hat{d}_{r e c-R F}\right)$ and $v(\alpha)$ :

$\alpha=\alpha+n_{\alpha}$

$\hat{d}_{r e c-R F},=\hat{d}_{r e c-R F}+n_{\hat{d}_{r e c-R F}} \hat{d}_{r e c-R F}=\hat{d}_{r e c-R F}+n_{\hat{d}_{r e c-R F}}$

where $n_{\hat{d}_{r e c-R F}}=N\left(0, v\left(\hat{d}_{r e c-R F}\right)\right)$ and $n_{\alpha}=N(0, v(\alpha))$.

The Root Mean Square Errors or RMSEs of the RF source, assuming noisy measurements for both the distance from the RF source to the receiver and the angle of arrival of the signal, are shown in Figures 3 and 4, respectively. In Figure 3, the distance from the RF source to the receiver is between 2 and $20 \mathrm{~km}$ (to mimic a real rescue mission). Furthermore, Figure 4 described the angle of arrival, in which it is assumed to be from 20 to $70^{\circ}$ (to prevent the full absorption of the signal due to the limited angle of reflection for RF signals, based on the reflector's type). For these measurements, Gaussian noises with 0 means and random standard deviations are applied, and the RMSE of each value is calculated after 100 runs. Figure 4 shows that, based on our proposed approach, the maximum RMSE when locating the RF source for a distance $20 \mathrm{~km}$ from the RF source to the receiver is $0.46 \mathrm{~km}$. 


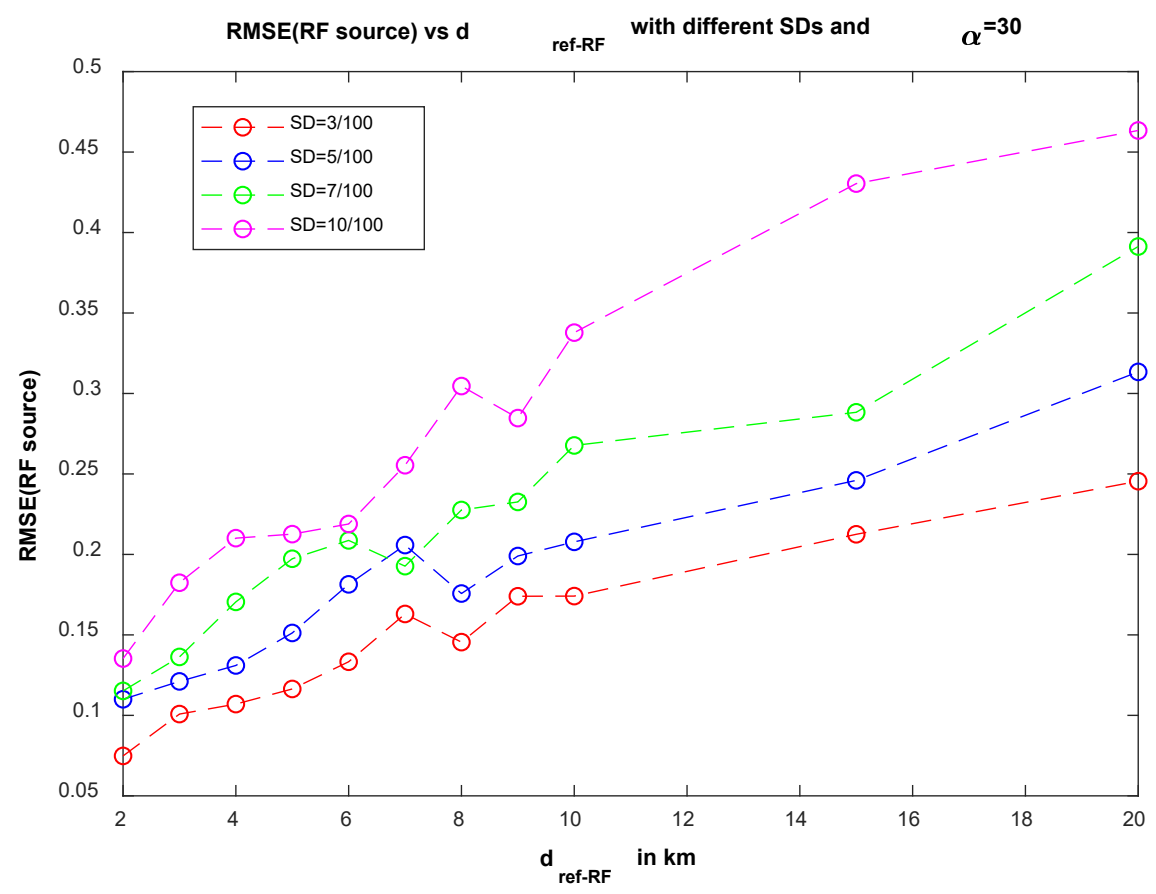

Figure 3 The RMSE of the RF source versus the distance from the RF to the receiver, assuming multiple standard deviations for different values of distances.

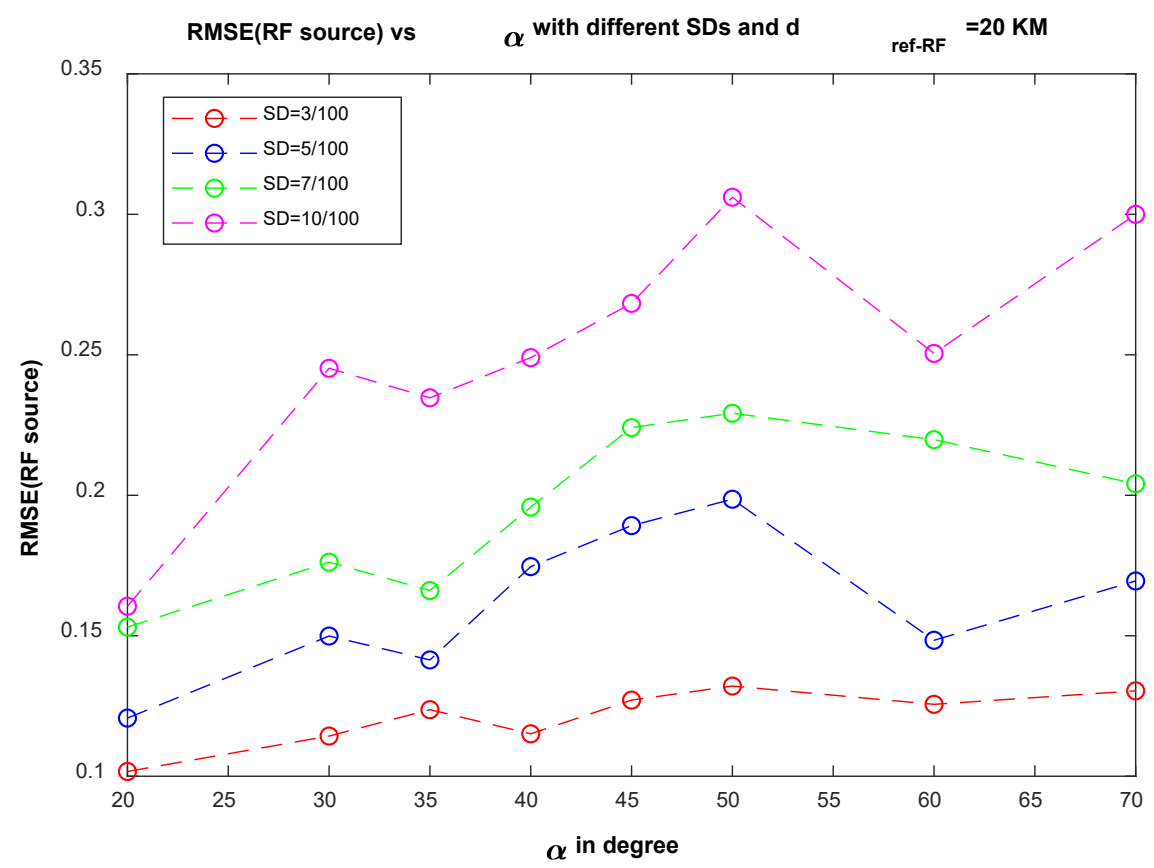

Figure 4 The RMSE of the RF source versus the angle of arrival of the RF's signal, assuming multiple standard deviations for different values of $\alpha$.

In Figure 4, the distance between the RF source and the receiver was fixed at $20 \mathrm{~km}$, but the angle of arrival of the RF source's signal was tested for different values and different standard deviations. The results show that although the distance is at the maximum $(20 \mathrm{~km})$, the RMSE of this case is lower than the case shown in Figure 3 (the maximum RMSE shown in Figure 4 is $0.3 \mathrm{~km}$ ), which means that the proposed approach is resistant to the noisy AOA measurements. 
Figures 3 and 4 show that although there exist noises while determining the AOA of the received signal, and the distance from the RF source to the receiver, the RF source and the reflector can be localized with good and acceptable precision in ambiguous areas, instead of unique positions.

A real scenario is proposed in Figure 5: Based on the RF source's signal, received from a lost person, the distance from the RF source to the receiver is calculated, $\hat{d}_{r e c-R F}=5 \mathrm{~km}$, and the angle of arrival of the RF source's signal is $\alpha=45^{\circ}$, when the receiver is sitting at the origin of the world frame.

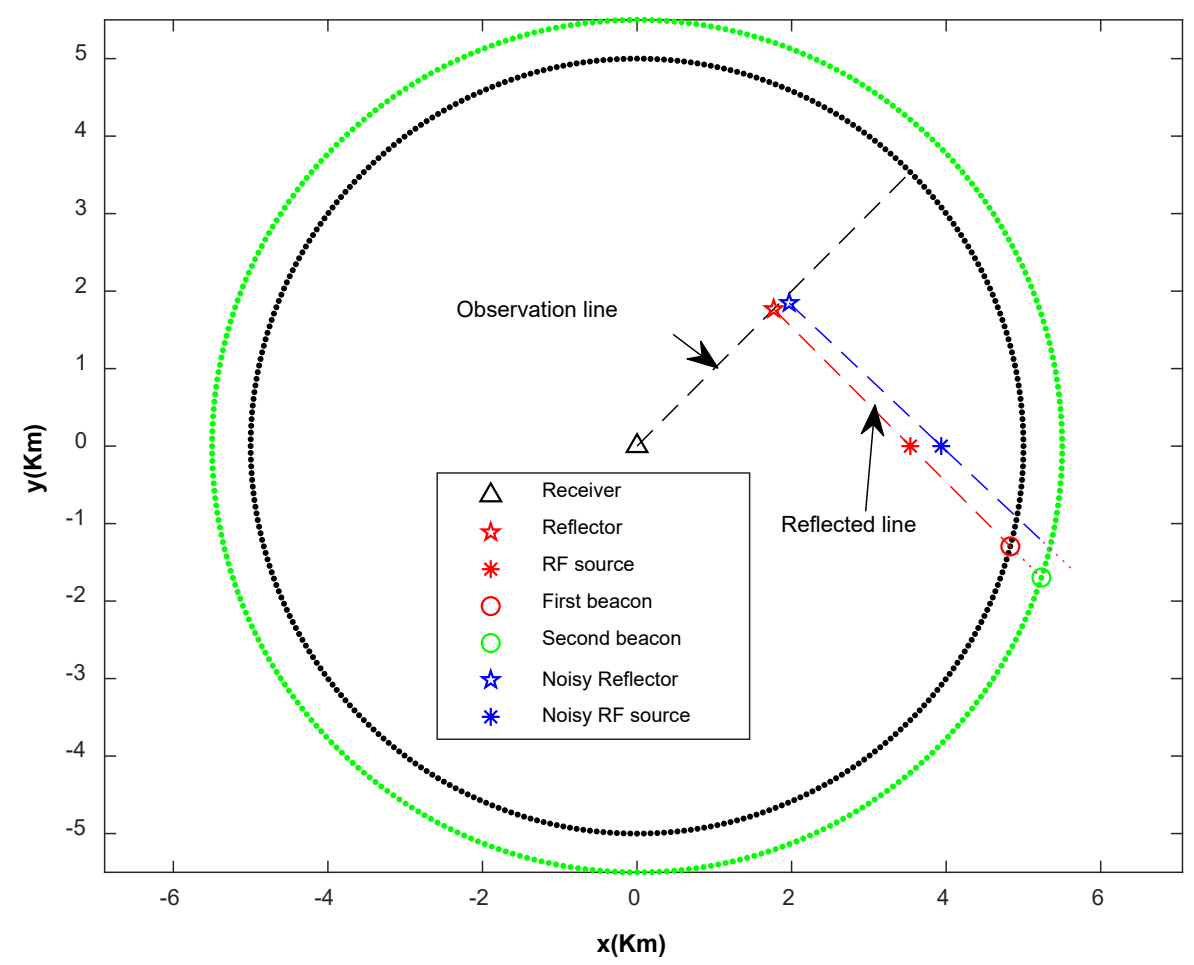

Figure 5 A scenario of finding an RF source using 2 beacons that move along the desired paths when the RF source's signal is received after 1-bounce reflection. The angle of arrival of the signal is $45^{\circ}$ and the distance calculated from the RF source to the receiver is $5 \mathrm{~km}$. The perimeter of the search space is shown as the black-dotted circle, and the determined position of the RF source (assuming noisy measurements) is shown as the blue star, instead of the red star.

As shown in Figure 5, it is clear that due to the noises in measurements, the direction of the reflected line (the line lying along the beacons' positions) shifted a little, and caused a drift in the position of the reflector. Thus, the position of the RF source, that depends on the reflector's orientation and position, is determined in an ambiguous area around its true position (the determined position of the RF source is nearly $100 \mathrm{~m}$ away from its true position, for a distance of $5 \mathrm{~km}$ from the RF source to the receiver), due to the error emanating from the localization of the reflector.

Table 2 Comparison of RMSE of our proposed approach against some methods introduced in other papers, for the same distances between the RF source and the receiver, in NLOS environment.

\begin{tabular}{lcc}
\hline \multicolumn{1}{c}{ Methods } & $\widehat{\boldsymbol{d}}_{\boldsymbol{r e c}-\boldsymbol{R F}} \mathbf{i n} \mathbf{~ k m}$ & $\mathbf{R M S E}\left(\widehat{\boldsymbol{X}}_{R F}\right)$ in $\mathbf{~ k m}$ \\
\hline Simultaneous target localization and obstacle [28] & $10-20$ & $1.18-1.49$ \\
Beacon-based method introduced in [5] & $9-15$ & $0.175-0.66$ \\
RSS/DOA combination method used in[6] & $3-10$ & $0.18-0.69$ \\
SLUS method introduced in [26] & 10 & 1.1016 \\
Our proposed approach & $3-20$ & $0.144-0.52$ \\
\hline
\end{tabular}


Table 2 shows a comparison in RMSE of the RF source position between different methods used for RF source localization, assuming the same distances between the RF source and the receiver for NLOS environment. The results show that our proposed approach has a lower RMSE for a distance up to $20 \mathrm{~km}$, with its performance validated through simulation.

\section{Conclusions}

In this paper, we introduced a novel beacon-based approach for localizing an RF source when its signal is received by a single stationary receiver in an NLOS environment after a 1-bounce reflection. The method relies on determining the search space in which the RF source is, and the search paths for the beacons to localize both the reflector and the RF source. By receiving the signals coming from the 2 beacons (that act as helpers, which can be elements of the Search and Rescue team) that move along determined paths, the reflector is localized, and the bearing of the RF source and its position are determined. Although some assumed parameters may have impacts on the localization approach, it is confirmed that our proposed approach is able to localize an RF source with an acceptable boundary of error. We evaluated the performance of our proposed approach in simulation, considering the RMSE of the RF source's position as the performance metric, and compared the results with other localization approaches. The presence of reflectors and obstructions between the RF source and the beacons should be analyzed in the future. Additionally, we intend to test the proposed approach in a real-world scenario to demonstrate its efficacy in search and rescue missions.

\section{References}

[1] R Severino and M Alves. Engineering a search and rescue application with a wireless sensor network-based localization mechanism. In: Proceedings of the 2007 IEEE International Symposium on a World of Wireless, Mobile and Multimedia Networks, Espoo, Finland. 2007, p. 1-4.

[2] P Kristalina, A Pratiarso, T Badriyah and ED Putro. A wireless sensor networks localization using geometric triangulation scheme for object tracking in urban search and rescue application. In: Proceeding of the $2^{\text {nd }}$ International Conference on Science in Information Technology, Balikpapan, Indonesia. 2016, p. 254-9.

[3] S Zorn, R Rose, A Goetz and R Weigel. A novel technique for mobile phone localization for search and rescue applications. In: Proceedings of the 2010 International Conference on Indoor Positioning and Indoor Navigation, Zurich, Switzerland. 2010, p. 1-4.

[4] MHA Meniem, AM Hamad and E Shaaban. Fast and accurate practical positioning method using enhanced-lateration technique and adaptive propagation model in GSM mode: Case study using android smart phone in Egypt roads. Int. J. Comput. Sci. Issues 2012; 9, 188-93.

[5] S Haidari, H Moradi and SM Dehghan. Improving localization in NLOS condition using RF beacons. In: Proceedings of the $4^{\text {th }}$ International Conference on Robotics and Mechatronics, Tehran, Iran. 2016, p. 272-7.

[6] S Haidari, H Moradi, M Shahabadi and SM Dehghan. RF source localization using reflection model in NLOS condition. In: Proceedings of the $4^{\text {th }}$ International Conference on Robotics and Mechatronic, Tehran, Iran. 2016, p. 601-6.

[7] AL Adams, TA Schmidt, CD Newgard, CS Federiuk, M Christie, S Scorvo and MD Freest. Search is a time-critical event: When search and rescue missions may become futile. Wilderness Environ. Med. 2007; 18, 95-101.

[8] Z Ma and K Ho. TOA localization in the presence of random sensor position errors. In: Proceeding of the 2011 IEEE International Conference on Acoustics, Speech and Signal Processing, Prague, Czech Republic. p. 2468-71.

[9] S Lee, W Lee and K You. TDOA based UAV localization using dual-EKF algorithm. In: D Slezak, TH Kim, A Stoica and BH Kang (Eds.). Control and automation. Springer, Berlin, Heidelberg, 2009, p. 47-54.

[10] HJ Du and JP Lee. Passive geolocation using TDOA method from UAVs and ship/land-based platforms for maritime and littoral area surveillance. Defence R\&D Canada, Ottawa, Canada, 2004.

[11] SMM Dehghan, MS Tavakkoli and H Moradi. Path planning for localization of an RF source by multiple UAVs on the Crammer-Rao lower bound. In: Proceedings of the 2013 First RSI/ISM International Conference on Robotics and Mechatronics, Tehran, Iran. 2013, p. 68-73.

[12] SMM Dehghan, H Moradi and SAA Shahidian. Optimal path planning for DRSSI based localization of an RF source by multiple UAVs. In: Proceedings of the $2^{\text {nd }}$ RSI/ISM International Conference on Robotics and Mechatronics, Tehran, Iran. 2014, p. 558-63. 
[13] C Cheng, W Hu and WP Tay. Localization of a moving non-cooperative RF target in NLOS environment using RSS and AOA measurements. In: Proceedings of the 2015 IEEE International Conference on Acoustics, Speech and Signal Processing, South Brisbane, QLD, Australia. 2015, p. 3581-5.

[14] W Xu, F Quitin, M Leng, WP Tay and SG Razul. Distributed localization of a RF target in NLOS environments. IEEE J. Sel. Area. Comm. 2015; 33, 1317-30.

[15] F Dai, Y Liu and L Chen. A hybrid localization algorithm for improving accuracy based on RSSI/AOA in wireless network. In: Proceedings of the 2012 International Conference on Computer Science and Service System, Nanjing, China. 2012, p. 631-4.

[16] G Ding, Z Tan, L Zhang, Z Zhang and J Zhang. Hybrid TOA/AOA cooperative localization in nonline-of-sight environments. In: Proceedings of the 2012 IEEE $75^{\text {th }}$ Vehicular Technology Conference, Yokohama, Japan. 2012, p. 1-5.

[17] C Wan, A Mita and S Xue. Non-line-of-sight beacon identification for sensor localization. Int. J. Distr. Sensor Network. 2012; 8, 459590.

[18] C Sha and RC Wang. A type of localization method using mobile beacons based on spiral-like moving path for wireless sensor networks. Int. J. Distr. Sensor Network. 2013; 9, 404568.

[19] N Rajagopal, S Chayapathy, B Sinopoli and A Rowe. Beacon placement for range-based indoor localization. In: Proceedings of the 2016 International Conference on Indoor Positioning and Indoor Navigation, Alcala de Henares, Spain. 2016, p. 1-8.

[20] M Alain and BH Irene. Indoor positioning through fingerprinting technics: How many beacons should be deployed and where? In: Proceedings of the $20^{\text {th }}$ International Symposium on Wireless Personal Multimedia Communications, Bali, Indonesia. 2017, p. 522-8.

[21] M Fares, H Moradi, M Shahabadi and S Nasiri. Target localization in NLOS condition using beacons to determine reflector orientation. In: Proceedings of the $6^{\text {th }}$ RSI International Conference on Robotics and Mechatronics, Tehran, Iran. 2018, p. 178-82.

[22] JJ Leonard and HF Durrant-Whyte. Mobile robot localization by tracking geometric beacons. IEEE Trans. Robot Autom. 1991; 7, 376-82.

[23] ML Sichitiu and V Ramadurai. Localization of wireless sensor networks with a mobile beacon. In: Proceedings of the 2004 IEEE International Conference on Mobile Ad-hoc and Sensor Systems, Fort Lauderdale, FL, USA. 2004, p. 174-83.

[24] MH Fares, H Moradi and M Shahabadi. SLTR: Simultaneous localization of target and reflector in NLOS condition using beacons, Available at: https://arxiv.org/abs/1911.03940, accessed January 2021.

[25] S Haidari, H Moradi and S Dehghan. RF source localization using obstacles map and reflections. Int. J. Ind. Electron. Contr. Optim. 2021; 4, 181-90.

[26] SMM Dehghan and H Moradi. SLAM-inspired simultaneous localization of UAV and RF sources with unknown transmitted power. Trans. Inst. Meas. Contr. 2016; 38, 895-907.

[27] P Pathania, P Kumar and BS Rana. Performance evaluation of different path loss models for broadcasting applications. Am. J. Eng. Res. 2014; 3, 335-42.

[28] SM Dehghan, S Haidari and H Mordi. Toward aerial simultaneous target localization and obstacle estimation using RSSI observations. In: Proceedings of the $3^{\text {rd }}$ RSI International Conference on Robotics and Mechatronics, Tehran, Iran. 2015, p. 517-22. 